\title{
Microencapsulation in food industry - an overview
}

\author{
Dorina Petkova ${ }^{1, *}$, Dasha Mihaylova ${ }^{1}$, and Ivelina Desseva ${ }^{2}$ \\ ${ }^{1}$ Department of Biotechnology, Technological Faculty, University of Food Technologies, Plovdiv, Bulgaria \\ ${ }^{2}$ Department of Analytical Chemistry and Physical Chemistry, Technological Faculty, University of Food Technologies, Plovdiv, \\ Bulgaria
}

\begin{abstract}
In the modern health concept, food quality is becoming more and more important. People are increasingly looking for added value to their diet through the presence of bioactive substances. Usually, the latter are sensitive molecules; they are unstable in processing and consumption. In addition, problems with unpleasant organoleptic characteristics clean labelling and high production costs can occur. To overcome these problems, a solution can be sought in microencapsulation techniques. Although these techniques have been known for a long time, nowadays their meaning and significance are gaining new dimensions. In this regard, this review aims to provide up-to-date information on currently used microencapsulation techniques, limitations, and prospects.
\end{abstract}

\section{Introduction}

Microencapsulation is a technology that is often used in the food industry because it has many useful applications. This technology is developing rapidly and is expressed in the fact that the target active compounds are incorporated into the wall materials, forming capsules protected from external influences. The wall materials used in the food industry are very different. Agents used for encapsulating materials in the food industry must have a number of properties such as being degradable, resistant to gastric juices, low viscosity, hygroscopicity and cost [1]. Having these properties, the wall materials manage to retain the active compounds and to be delivered to the target site and protected from adverse environments [2, 3]. The encapsulation technique is used in many other fields besides the food industry. Some of them are medicine, agronomy and pharmacy. In the diet it is usually used for microencapsulation of essential oils, colorants, flavors, sugars, microorganisms and others [4]. In order to create foods that, in addition to their nutritional value, also have substances that are beneficial to human health, the microencapsulation technique is used. Such foods have elevated levels of calcium, for example, which serves to prevent osteoporosis or microorganisms that produce lactic acid to lower cholesterol. Another example is the addition of phenolic compounds to combat heart problems [5].

Encapsulation is mainly used to improve the bioavailability and absorption through the cells, to control the release of the base material, as well as its targeted delivery, concealment of unpleasant tastes and odors, easier handling and storage.
The encapsulated material may be antioxidants, polyphenols, flavors, colors, preservatives, sweeteners, proteins, minerals, lipids, probiotics, etc. [6].

Microencapsulation is used in the food industry mainly to achieve greater stability of the active substance that is encapsulated. Food processing is associated with many environmental changes, such as heat treatment, cooling, freezing and more. These changes can adversely affect the capsules and make them unstable [7].

This process produces products called nano- or microcapsules, which can be nano- or microspheres. The internal structure and morphology of the nano- and microcapsules serve to distinguish them.

Depending on the size of the capsules, they can be divided into microcapsules, microspheres or microparticles, with a diameter between 1-800 $\mu \mathrm{m}$, and nanoparticles, nanospheres or nanocapsules with a diameter below $1 \mu \mathrm{m}$, respectively $[8,9]$.

The use of microencapsulation in the food industry and its benefits have been extensively studied $[10,11,12$, $13,14,15]$. The reasons why encapsulation techniques are used are numerous, here are some of them: (1) active compounds that are sensitive to external conditions to be protected from them; (2) to protect volatile compounds from straightening; (3) to disguise unpleasant aroma and taste; (4) to facilitate the use of liquids by turning them into a solid form; (5) for higher solubility of materials that do not dissolve well; (6) to obtain delivery of active substances to a specific site and their controlled release; and (7) to improve the efficacy of bioactive compounds $[10,15,17,18,19]$.

* Corresponding author: dorina.petkova95@gmail.com 


\section{Materials for microencapsulation}

The stability, size and properties of the capsules depend on the encapsulation methods and materials used. Antioxidants, probiotics, dyes, minerals, vitamins, oils, flavors, microorganisms and many others are used as encapsulated materials in the food industry $[10,11,17]$.

\subsection{Wall materials}

Wall materials serve to protect the base material. Their choice is based on the characteristics of the base material, as well as the physicochemical properties of the wall material, such as molecular weight, emulsifying properties, solubility, film-forming properties [49]. The wall materials used in the food industry must meet a number of criteria, such as being safe, biodegradable and not affecting the taste of the food. [50,51].

Both natural and synthetic polymers can be used in food products. Natural polymers are used more, and they can be polysaccharides such as cellulose and its derivatives, starch, animal and microbial extracts, as well as plant extracts and exudates [52], as well as plant and animal proteins. Synthetic polymers used as encapsulating agents include polybutadiene, polylactic acid, polystyrene, polyvinyl acetate, polypropylene, and the like [53].

In the encapsulation of nutrients as wall materials are used: carbohydrates (cyclodextrins, starch, maltodextrin); proteins (gelatin, soy and whey protein); hydrocolloids (chitosan, pectin, alginate, agar, gum arabic); cellulose and lipids (wax, paraffin, phospholipids) [11].

The most commonly used wall materials in the food industry are gums. They exist naturally in nature, in plants, but are also synthesized by bacteria. Gums are a group of polysaccharides and their most famous representative is gum arabic. It has a low viscosity in water and retains over $85 \%$ volatile compounds. Another property of encapsulating aromatic compounds is that it protects essential oils and volatile components from oxidation [54].

Proteins used as a wall film are used for encapsulation by coacervation. Protein film is a great barrier to oxygen and unwanted odors [55]. Figure 1 schematically describes the materials most commonly used as wall and core materials.

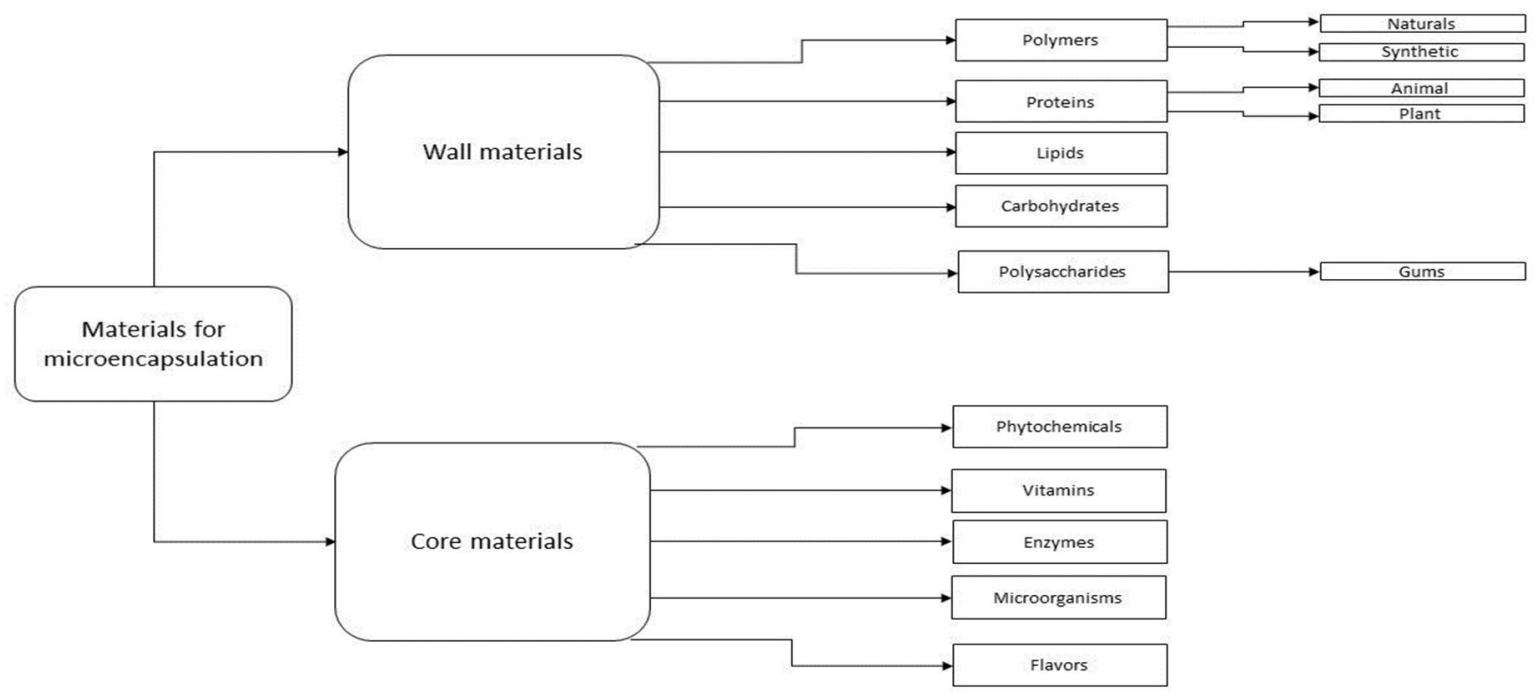

Fig. 1. Schematic representation of the materials used in the encapsulation process

\subsection{Core materials}

The materials that are in the core of the capsule are the active components that are encapsulated in the wall material. They can be liquid, solid or gas. The nature of the product obtained in the end depends on the properties of the materials used for encapsulation. Depending on how the basic materials are arranged, single-core and multi-core capsules can be obtained. Single-core capsules represent a single continuous phase of core material in the wall material, and multi-core capsules represent multiple units of base material in the wall material. There are also matrix-type capsules in which the base material is homogeneously distributed in the wall material [6].

\subsubsection{Microencapsulation of phytochemicals}

Phytochemicals are chemical compounds that are found in plants and have biological activity. Studies on them show that they have a good effect on human health and serve as disease prevention. They have antibacterial, antioxidant, antiviral, anti-inflammatory and anti-cancer properties, as well as regulate blood pressure, cholesterol synthesis and stimulate the immune system $[56,57,58]$.

The incidence of age-related illnesses and chronic diseases such as stroke, cancer and heart disease decrease with higher consumption of fruits and vegetables rich in phytochemicals $[59,60,61,62]$.

Phytochemicals are found mainly in vegetables, fruits, whole grains and legumes. What will be the content of phytochemicals and where they will be distributed in 
the plants depends on several factors - the time in which the crop is harvested, the variety and type of plant and how the crop is processed and stored [60].

In the last few decades, interest in phytochemicals has continued to grow, driven by the fact that they improve health and prevent disease. They are increasingly found in various fields such as nutrition, medicine, pharmacy, and cosmetics.

One of the applications of phytochemicals is as natural flavors, aromatizers and food colors. Some of them are responsible for the colors of fruits and vegetables. Physical and chemical methods, such as cold pressing and extraction, are used to extract phytochemicals from plants [58].

Resveratrol, found in red grapes, peanuts and mulberries, is a natural polyphenol that is difficult to dissolve in water. It is also easily oxidized and isomerized from the trans to the cis isomer upon exposure to light. In this case, encapsulation helps to direct the supply of resveratrol and protect the compound from external conditions [63].

\subsubsection{Microencapsulation of Vitamins}

Vitamins are essential for human health. They are essential nutrients and facilitate the treatment of skin problems and the regulation of oxidative stress in the body. Vitamins are easily oxidized, which makes them unstable. Improving their physicochemical stability, as well as increasing their physiological strength, is accomplished by nano- and microencapsulation [64].

Vitamin A, which is derived from $\beta$-carotene and has antioxidant functions and serves as a colorant, is a fatsoluble vitamin [65] and can be extracted from fish oil, dairy products or the liver. Its precursor is b-carotene, which promotes the body's natural immunity, cell growth, and promotes bone growth and visual health [66]. It is naturally found in plants, unlike $\beta$-carotene. $\beta$-carotene can be found in mango, pumpkin, carrot and others [67]. However, both water- and fat-soluble vitamins could be microencapsulated $[68,69,70]$.

In this aspect, as early as 2001, Junyaprasert et al. investigate how the encapsulation of vitamin A palmitate is affected by coacervation of gelatin and acacia. The studies describe the influence of the concentration of the solution in the core, the hardener, the core-wall ratio, the method of dissolution and the colloidal mixing ratio [71].

\subsubsection{Microencapsulation of Enzymes}

Enzymes have protein properties and therefore can affect the speed and specificity of reactions in the human body and food. They are catalysts and the reactions associated with them are inextricably linked to the temperature at which the reaction takes place, as well as $\mathrm{pH}$ and ionic strength. Given that the enzymes have a protein character, high temperature and inappropriate $\mathrm{pH}$ lead to denaturation. This can be prevented by applying encapsulation techniques to the enzymes. This preserves their stability during processing and storage, as well as avoids unwanted interactions with other compounds in the food matrix [72].
The presence of detergents adversely affects the enzymes, causing structural changes in its binding site. This reduces the activity, but this can be prevented by encapsulating the enzymes. The encapsulated enzyme shows a change in optimal operating conditions, maintains full catalytic activity and has better temperature stability than the free enzyme [73].

Encapsulated ligninolytic enzymes are used in the production of juices and more precisely in their clarification. For this process, the enzymes are encapsulated and thus have a higher activity in the clarification and removal of polyphenolic compounds [74].

\subsubsection{Encapsulation of Microorganisms}

Probiotic bacteria, taken in appropriate amounts, have been shown to have a good effect on human health and that foods that contain live microorganisms support the immune system and prevent certain diseases [75]. When there are not enough viable microorganisms, the health benefits are minimal because they are not delivered to the body. If physical protection of living probiotic microorganisms from the environment and adverse conditions is created, they will retain their properties. Encapsulation of probiotics plays an important role in their functionality in food and their viability. Also, by encapsulating them, the desired effect is achieved, they are released in certain parts of the intestine and thus increase their health benefits. The most commonly used techniques for encapsulating probiotics are spray drying, emulsification and extrusion [76].

\subsubsection{Encapsulation of Flavors}

The aroma of food is essential and is closely related to how a food is perceived by consumers. Aromatic compounds depend on the temperature of processing and storage and are therefore difficult to control because they are volatile. Encapsulation techniques are used to protect odors from evaporation during processing and storage. Depending on the wall materials used and the processing conditions, the volatile compounds can be encapsulated in various forms [23].Oleoresins are highly sensitive to light, oxidation and heat. Encapsulation techniques are used to prevent this sensitivity. Cardamom oleoresin, for example, is encapsulated using gum arabic, modified starch, or maltodextrin for encapsulating materials, and then spray drying is used [77].

Orange oil is sensitive to oxygen, and whey protein isolates are used to build a barrier, which provide an effective basis for trapping volatiles by spray drying [78].

\section{Techniques for microencapsulation}

The choice of encapsulation technique directly determines the nature of the wall and core material, the desired particle size, the release mechanism, and the encapsulation efficiency. The classification of encapsulation techniques is according to their nature, namely chemical or physical. Techniques that belong to the chemical are polymerization and polycondensation. Physical techniques are divided into physicochemical and 
physico-mechanical. Physicochemical encapsulation techniques are coacervation, sol-gel and supercritical microencapsulation with $\mathrm{CO}_{2}$ and physico-mechanical spray drying, liquid coating, centrifugal encapsulation, vacuum encapsulation and electrostatic encapsulation [20].

Another type of classification that can be used for encapsulation techniques is according to the medium used as a suspension. A liquid medium is used to dissolve the active substance in the following techniques: coacervation, emulsification, solvent evaporation, in situ and interphase polymerization. Techniques that use gas as a medium are spray drying, spray cooling / cooling, fluidized bed coating [17].

\subsection{Physico-mechanical process}

\subsubsection{Spray-drying}

Spray drying is an encapsulation method used to produce dry products from liquid materials. This process is fast, continuous, cost-effective, and easy to scale. It is expressed in that the liquid in which the base material is dissolved is sprayed by means of a spray in a hot drying gaseous medium, usually air. The droplets that form during spraying lose the solvent in the drying chamber, resulting in solid particles, which are subsequently removed from the air stream and collected. Spray drying is often used in the food industry to reduce water content and ensure microbiological stability of products. This drying technique is used to encapsulate nutrients such as flavors, lipids, and carotenoids [21, 22].

Each food has its own characteristic aroma, which is due to the aromatic compounds in it. However, these compounds are highly volatile and because of this, they are often lost during spray drying. The composition of the wall material, how it affects the microencapsulation, and the controlled release of the encapsulated aromas has been studied many times [23]. A good example of how to preserve the aromatic compound is the encapsulation of L-menthol by spray drying. This compound is a cyclic terpene alcohol, occurring mainly in the form of crystals or granules with a melting point of $41-43^{\circ} \mathrm{C}$, which shows high volatility and whisker growth [24]. These barriers limit the use of L-menthol in the food industry and its shelf life. Therefore, they are overcome by encapsulation by spray drying. Other flavors that have been successfully encapsulated by spray drying are those of citronella, marjoram, and oregano. They are encapsulated in wall materials of skimmed milk powder and whey protein concentrate [25].

\subsubsection{Freeze-drying}

Freeze-drying or lyophilization is a simple and easy way to encapsulate [26]. This method is used for materials that are sensitive to high temperatures, such as volatile compounds, as the removal of moisture takes place by freezing the sample and then evaporating the moisture at low temperatures under vacuum, as opposed to spray drying, which is performed at high temperatures [11].
Unprofitability and the need for a lot of time for dehydration are only some of the disadvantages of this encapsulation method, which in turn is the reason why this method is used less often for encapsulation of phytochemicals, compared to spray drying. Powdered products obtained after lyophilization have a porous structure and need to be ground into small particles after drying. In some cases, the particle size changes and the encapsulated material may protrude beyond the capsule to the surface of the particles. Lyophilization as an encapsulation technique has a number of disadvantages, but it is nevertheless often used and is suitable for storing and encapsulating many compounds [27].

Lyophilization as an encapsulation technique is used in the food industry and as an example of its application is the encapsulation of limonene. It is one of the most important aromatic compounds in citrus fruits and has positive effects on humans. In 2007, Kaushik and Roos managed to encapsulate limonene by freeze-drying [28]. This encapsulation technique is most commonly used in active compounds to achieve their controlled release [29] as well as to recover by-products from agro-industrial processes. In addition, gum arabic, xanthan gum, sodium alginate and soy protein-like wall materials were used for microencapsulation of carotenogenic extracts by a lyophilization method [30]. Gum arabic and maltodextrin were used as coating materials for probiotic carrot juice powder [31].

\subsubsection{Extrusion}

This encapsulation technique is mainly used for volatile and unstable flavours in glassy carbohydrate matrices. One of the most important advantages of this method is that prone to oxidation aromatic compounds acquire a long shelf life after encapsulation. Atmospheric gases pass very slowly through the glass matrix, which is hydrophilic, thus providing an almost impermeable barrier against oxygen. Carbohydrate matrices in glass states have very good barrier properties and extrusion is a convenient process that allows the encapsulation of flavours in such matrices [32].

Encapsulation of $\beta$-cyclodextrin by extrusion has been shown to be an effective way to protect limonene from oxidation [33]. Most of all extrusion is used for encapsulation of Lactobacillus strains [34] normally resulting in increasing survival rate of the strains.

\subsubsection{Fluid Bed Coating}

The fluidized bed coating is a batch process in which the coating is applied to solid dust particles. This encapsulation technique is performed at a certain temperature, as the solid particles and the wall material are mixed by air flow. The material used for the shell must have a number of properties, such as being thermally stable, not interfering with injection and pumping, which depends of its viscosity, and to allow the formation of a film on the surface of the particles. The wall materials used in fluidized bed encapsulation are usually aqueous solutions of starch and cellulose derivatives, proteins, dextrins and resins. The evaporation of water from the 
aqueous solution used for encapsulation depends on many factors, such as the temperature of the solution, the spray air and the base material in the chamber. Other factors on which evaporation depends are the water content in the solution of the coating material, the speed of its dispersion, the humidity of the inlet and outlet of the chamber and the air flow. In the central flow of the inner column, the air flow rate is about $80 \%$ and $20 \%$ in the periphery, which leads to the circulation of dust particles. These characteristics of the method reduce agglomeration and increase the drying rate [35].

The fluidized bed encapsulation is used for many ingredients that are contained in much improved foods, and dry mixes. This technique encapsulates many food additives, such as lactic, citric, and ascorbic acid, as well as leavening agents such as sodium bicarbonate for pastries. The applicability and advantages of fluidized bed coating and other techniques for encapsulation in food are well known [36]. The technique is broadly used for probiotic bacteria to improve the survival [37].

\subsection{Chemical methods}

\subsubsection{Interfacial polymerization (IFP)}

In this encapsulation technique, the wall material used for encapsulation can be formed on the surface of the droplets, using polymerization of reactive multifunctional monomers. The most commonly used monomers in this method are multifunctional acid chlorides and isocyanates and it is possible to use them in combination or separately. The monomer used is dissolved in a liquid core material and distributed evenly in the aqueous phase, which contains a dispersing agent. Then a co-reactant multifunctional amine is added which leads to rapid polymerization at the interface generating the capsule shell [38]. Thus, different interactions form different types of shells, for example, a polyurethane shell will be formed by reacting the isocyanate with a hydroxylcontaining monomer, and by reacting the acid chloride with an amine, a polynylon or polyamine shell will be generated $[38,39]$.

\subsubsection{In situ polymerization}

This encapsulation technique is similar to IFP in terms of capsule formation. Here, too, it is formed by polymerization of monomers, which are placed in the apparatus where encapsulation occurs. In this technique there is no reactive agent introduced into the material to be encapsulated, the polymerization is carried out exclusively in the continuous phase and on the side of the continuous phase of the interface formed by the dispersed material of the core and the continuous phase. The polymerization of the reagents located there generates a low molecular weight prepolymer and over time this prepolymer increases, it is deposited on the surface of the encapsulated dispersed material from the core, where the crosslinking polymerization continues, thus obtaining hard capsule shells [40].

High speed mixers are used to obtain an emulsion with the desired droplet size and to make it stable. It is also important to emulsify the oil phase in water with watersoluble polymers, it is necessary to lower the $\mathrm{pH}$, to add melamine resin, which is water-soluble, and to initiate polycondensation, which gives crosslinked resins, which are deposited at the boundary between oil droplets and the aqueous phase. When the wall material hardens, capsules of micro size are formed and an aqueous dispersion of oil droplets is obtained. This method is used in ureaformaldehyde and melamine-formaldehyde encapsulation systems [41].

\subsection{Physico-chemical process}

\subsubsection{Coacervation}

A technique in which the base material is mixed with the wall material and deposited on the surface of the base material after phase separation is called coacervation. Phase separation is classified into simple and complex coacervation, as in simple coacervation the separation is due to desolvating (coacervating) agents, and in complex - to oppositely charged polymers. After phase separation, the particles must be stabilized by means of a suitable crosslinking reagent or by heat treatment [19]. The nature of the polymers and their interaction are important for the final shape and size of the capsule [42].

Coacervation has many advantages. Some of them are high encapsulation efficiency (about 90\%), high temperature is not required, the obtained capsules are not thermolabile, the use of organic or toxic solvents is not required. The main disadvantage of this technique is that glutaraldehyde is used as a crosslinking reagent, and this does not allow its use in the food industry. This disadvantage can be overcome using enzymes or natural crosslinking agents such as genipin, anions, polysaccharides, and proteins [2, 43, 44, 45].

Coacervation encapsulation is applied to some phytochemicals. This is the case, for example, with capsaicin, which is found in hot peppers and is responsible for their peppery taste. Capsaicin is widely used in the food industry, being most used as a flavoring of spicy dishes [46].

\subsubsection{Polymer encapsulation by rapid expansion of supercritical fluids}

Supercritical liquids have the beneficial properties of both liquids and gases and are highly compressed gases. The most commonly used supercritical fluids are nitric oxide $\left(\mathrm{N}_{2} \mathrm{O}\right)$, alkanes $\left(\mathrm{C}_{2}\right.$ to $\left.\mathrm{C}_{4}\right)$ and carbon dioxide $\left(\mathrm{CO}_{2}\right)$. A very slight change in pressure or temperature leads to a change in density close to the critical point of the fluids. The most commonly used encapsulation fluid is supercritical carbon dioxide because it is readily available and cost-effective, has a low critical temperature, has no toxic and flammable properties, and has a high purity [47]. The most commonly used methods are rapid supercritical expansion; gas anti-solvent; and particles of gas-saturated solution [48, 47]. 


\section{Application of microencapsulated materials to food packaging}

The properties possessed by micromaterials have been used to develop structures that have properties such as lower gas permeability, improved thermal stability and strength, and others. These properties are more pronounced in the already formed structures than in the single structure of the material. To increase the shelf life of a product, micro-materials are already used in packaging systems as carriers for the delivery of active compounds. Active packaging not only serves to package the product, but also has improved protective properties. These properties are expressed in the release of antimicrobial compounds, change in gas leakage, removal of oxygen from the inside of the package and others.

When a package is equipped with internal or external indicators that provide information about the actual quality and / or history of the product and thus improves the communication aspect of the package, it is called smart packaging [79].

Food sold on the market must meet the expectations of buyers, be fresh and quality. If these expectations are not met, it affects the sales and image of companies, and on the other hand, if you inadvertently consume expired food, there is a danger of food poisoning. The expiration date printed on the packaging does not take into account potential physical, thermal and / or microbiological changes in the product, so this approach is not the best to avoid selling expired products. In recent years, food manufacturers have begun to use modern packaging systems to avoid problems with the suitability of the food product.

In order to obtain accurate information about the product and whether it is high quality and safe, packaging has been developed that interacts dynamically with the food product, called smart packaging [80].

The active packaging, in addition to preventing the product from interacting with external conditions, has other important properties, such as - control of surface pollution, which is mainly related to microbial pollution, protects products such as fish and seafood, which have high water activity [81]. The use of active packaging allows to reduce the use of preservatives or to simplify the production process by adding preservatives in the packaging stage, and thus enhance the organoleptic properties [82]. Such packaging systems reduce the inactivation of substances [83], as well as carry out controlled penetration of controlled bacteriostatic active substances [84].

The antimicrobial activity carried out by the active packages is due to the fact that they successfully include additives having the activity. Such additives are replaced by silver zeolite [85], organic acids and their salts, bacteriocin such as nisin and pediocin [86], enzymes such as lysozyme [87, 88], chelators such as EDTA and lactoferrin $[89,90]$, the organic compound triclosan [82], plant extracts [91], chitosan [92] and a combination of several of the above as barrier technology [93].

\section{Bioactivity of microencapsulated ingredients}

Much attention is paid to functional foods and nutrients and more precisely to the systems that serve for delivery and are based on nanomaterials. They improve the delivery of active substances to a specific place, as well as improve absorption and bioavailability in the body [94, 95]. By ingesting nutrients orally, they are subjected to severe conditions such as low $\mathrm{pH}$, gastrointestinal (GI) barriers and food-tar enzymes, and thus their bioactivity and functionality are compromised. In addition to the conditions to which they are subjected, another disadvantage of functional constituents is that their solubility in water and body fluids is often low, and some oral neutraceuticals tend to be rapidly metabolized in the intestine and liver, which are closely related to low absorption, as well as with rapid clearance from the body.

The challenge is, therefore, to overcome these obstacles and move efficiently across the GI tract, finally reaching the systemic circulation without significant loss of bioactivity. Given that nanoparticles easily penetrate the plasma membrane, due to their very small size, it can be concluded that the encapsulation of bioactive molecules with nanomaterials will help their solubility in water, their ability to penetrate the gastrointestinal tract and their physicochemical stability [7, 96, 97].

\section{Innovations and challenges for microencapsulation in the food industry}

Microencapsulation technology was an innovation at the time of the first such development. Microencapsulation technologies are widespread, but there are industries in which there is room for innovation in this area [98]. Research is constantly evolving in terms of characterizing microencapsulation, with scientists constantly looking for new techniques, materials, and methods. This is necessary to overcome the challenges facing the food industry [99]. Innovations in the materials used for microencapsulation in the food industry are more difficult to achieve due to the restrictions imposed by regulatory authorities. However, some nutrients after processing can be used to encapsulate active compounds and nutrients [6].

Nowadays, human nutrition is a very delicate area and more and more new types of diets are entering. They are inextricably linked with challenges, but also opportunities for microencapsulation. Many food groups, such as gluten, carbohydrates, dairy products, lactose, sugar and fats, are being avoided due to consumer intolerance and in line with the trend of pure labeling [100]. Many of the proteins used for wall materials in encapsulation are animal-based. This will need to change over time, as additional pressure is expected to reduce the use of animal products [101, 102]. Plant proteins are already widespread [103]. Pea, wheat, and soy proteins are often used as wall materials. Barley and maize prolamins are used to polymerize the wall material [105]. In general, one of the main challenges for encapsulation in the food industry is the limited range of suitable encapsulating materials permitted for use in food [106]. 
Requirements for the purity and use of wall material vary from country to country, so it must meet food standards in the producer country and the exporting country [107].

\section{Conclusion}

Given all the advantages and disadvantages of microencapsulation techniques, it can be concluded that these technologies will continue to evolve. On the one hand, new techniques or materials will be sought to overcome the negative qualities.

Despite the disadvantages of the techniques used, the multiple advantages cannot be ignored. Microencapsulation allows the use of environmentally sensitive compounds in human nutrition, and thus they favor human health. In summary, we can say that the techniques for microencapsulation of substances and their use in the food industry is a very promising area that will continue to develop.

\section{References}

1. F. Barros, P. Stringheta. Biotecn. Cien. Desenvol. 36, 18-24 (2006)

2. S. Gouin. Trends Food Sci. Technol. 15, 330-347 (2004)

3. Y.K. Lee, M.A. Mijan, P. Ganesan, H.S. Kwak, Int. J. Dairy Technol. 66, 417-423 (2013)

4. H.M.C. Azeredo, Alimen. Nutrição. 16, 89-97 (2005)

5. P. Sanguansri, M.A. Augustin, Trends Food Sci. Technol. 17, 547-556 (2006)

6. C. Anandharamakrishnan, S.P. Ishwarya. Spray drying techniques for food ingredient encapsulation (Wiley Blackwell, John Wiley \& Sons Ltd., Hoboken, NJ, 2015)

7. D.J. McClements, E.A. Decker, Y. Park, J. Weiss, Crit. Rev. Food Sci. Nutr. 49, 577-606 (2009)

8. C. Thies, Microincapsulation - methods and industrial applications (Marcel Dekker Inc., Ney York, 1996)

9. M.J. Alonso, C. Remunan, Tecnología Farmacéutica Aspectos fundamentales de los sistemas farmacéuticos y operaciones básicas. II. (Sintesis S.A., Madrid, 1997)

10. J.D. Dziezak, Food Technol. 42, 136-151 (1988)

11. F. Shahidi, X.Q. Han, Food Sci. Nutr. 33, 501-547 (1993)

12. C. Schmitt, C. Sanchez, S. Desobry-Banon, J. Hardy, Food Sci. Nutr. 38, 689-753 (1998)

13. B.F. Gibbs, S. Kermasha, I. Alli, C.N. Mulligan, Food Sci. Nutr. 50, 213-224 (1999)

14. Z.X. Fang, B. Bhandari, Trends Food Sci. Technol. 21, 510-523 (2010)

15. S.S. Kuang, J.C. Oliveira, A.M. Crean, Crit Rev Food Sci. Nutr. 50, 951-968 (2010)

16. M. Fathi, M.R. Mozafari, M. Mohebbi, Trends Food Sci. 23, 13-27 (2012)

17. P.M.M. Schrooyen, R. Van Der Meer, C.G. De Kruif, Proc. Nutr. Soc. 60, 475-479 (2001)

18. R. Dubey, T.C. Shami, K.U.B. Rao, Def. Sci. J. 59, $82-95$ (2009)
19. N.V.N. Jyothi, P.M. Prasanna, S.N. Sakarkar, K.S. Prabha, P.S. Ramaiah, G.Y. Srawan, J. Microencap. 27, 187-197 (2010)

20.S.K. Ghosh, Functional coatings: by polymer microencapsulation (John Wiley \& Sons Ltd., Hoboken, NJ, 2006)

21. S.M. Razi, A. Rashidinejad, Spray drying encapsulation of bioactive materials (CRC Press, Boca Raton, 2021)

22. Z. Akbarbaglu, S.H. Peighambardoust, K. Saraband, S.M. Jafari. Food Chem. 359, 129965 (2021)

23. A. Madene, M. Jacquot, J. Scher, S. Desobry, Int. J. Food Sci. 41, 1-21 (2006)

24. A. Soottitantawat, K. Takayama, K. Okamura, D. Muranaka, H. Yoshii, T. Furuta, T, IFSET. 6, 163-170 (2005)

25. R. Baranauskiené, P.R. Venskutonis, K. Dewettinck, R. Verhé, Int. Food Res. J. 39, 413-425 (2006)

26. P. Laine, P. Kylli, M. Heinonen, K. Jouppila, J. Agric. Food Chem. 56, 11251-11261 (2008)

27. V. Marcillo-Parra, D.S. Tupuna-Yerovi, Z. González, J. Ruales, Trends Food Sci. 116, 11-23 (2021)

28. V. Kaushik, Y.H. Roos, LWT - Food Sci. Technol. 40, 1381-1391 (2007)

29. N. Garti, D.J. McClements, Encapsulation technologies and delivery systems for food ingredients and nutraceuticals (Woodhead Publishing, Elsevier, Sawston, 2012)

30. L. T. Braga, C. M. Borba, T. V. D. Rodrigues, C. C. Moraes, J. F. M. Burkert, Braz. J. Dev. 7, 70051$70066(2021)$

31. D. Rishabh, A. Athira, R. Preetha, J. Food Sci. Technol. 58, 05259-2 (2021).

32. D. Zasypkin, M. Porzio, J. Microencap. 21, 385-397 (2004)

33. S. Yuliani, P.J. Torley, B. D'Arcy, T. Nicholson, B. Bhandari, Int. Food Res. J. 39, 318-331 (2006)

34. H. Mirzaei, H. Pourjafar, A. Homayouni, Food Chem. 132, 1966-1970 (2012)

35. N. J. Zuidam, V. Nedovic, Encapsulation technologies for active food ingredients and food processing (Springer, New York. 2010)

36. R. G. Szafran, Nanotechnol. 3, 71-105 (2013)

37. L. Zaghari, A. Basiri, S. Rahimi, Int. J. Food Eng. 16, 20190384 (2020)

38. H. B. Scher, Microencapsulation of pesticides by interfacial polymerization: process and performance considerations (Pergamon Press, Oxford,1983)

39. D. Saihi, I. Vroman, S. Giraud, S. Bourbigot, React. Funct. Polym. 66, 1118-1125 (2006)

40. M. Cakhshaee, R.A. Pethrick, H. Rashid, D.C. Sherrington, Polymer. 26, 185-192 (1985)

41. G. Ano, A. Esquisabel, M. Pastor, A. Talavera, B. Cedré, S. Fernández, S. Sifontes, Y. Aranguren, G. Falero, L. García, R. Lydia Solís, J. Luis Pedraz, Vaccine. 29, 5758-5764 (2011)

42. Y. Lv, F. Yang, X. Li, X. Zhang, S. Abbas, Food Hydrocoll. 35, 305-314 (2014)

43. C. Dima, M. Cotârlet, P. Alexe, S. Dima, Innov. Food Sci. Emerg. Technol. 22, 203-211 (2014) 
44. P.N. Ezhilarasi, P. Karthik, N. Chhanwal, C. Anandharamakrishnan, Food Bioprocess Technol. 6, 628-647 (2013)

45. Y. Yin, J. Li, Chitosan-based hydrogels: functions and applications (CRC Press, Boca Raton, 2012)

46. K. Srinivasan, Food Rev. Int. 21, 167-188 (2005)

47. I.H. Ya, C. Yu-Hsiu, Y. Chien-Chih, T. Tong-Rong, C. Thau-Ming, Colloids Surf. B. 58, 290-297 (2007)

48. K. Matsuyama, K. Mishima, K.I.H. Hayashi, J. Matsuyama, Nanophysics and nanotechnology: an introduction to modern concepts in nanoscience (John Wiley \& Sons, Inc., Hoboken, 2003)

49. A. Gharsallaoui, G. Roudaut, O. Chambin, A. Voilley, R. Saurel, Food Res Int. 40, 1107-1121 (2007)

50. M.A. Augustin, Y. Hemar, Chem. Soc. Rev. 38, 902912 (2009)

51. M. Fathi, Á. Martín, D.J. McClements, Food Sci. Technol. 39, 18-39 (2014)

52. C. Wandrey, A. Bartkowiak, S.E. Harding, Encapsulation Technologies for Active Food Ingredients and Food Processing (Springer, New York, 2010)

53. J.M. Lakkis, Encapsulation and controlled release technologies in food systems (Wiley, Hoboken, 2016)

54. L.C. Aburto, D.Q. Tavares, E.T. Martucci, Ciênc. Tecnol. Aliment. 18, 45-48 (1998)

55. L.C. Conto, G.D. Fernandes, C.R.F. Grosso, M.N. Eberlin, L.A.G. Gonçalves. Int. Food Res. J. 54, 432438 (2013)

56. A.J. Day, J.A. Rothwell, R.A. Morgan, Phytochemicals in health and disease (Marcel Dekker, Inc New York, 2004)

57.D. Herber, Herbal medicine: biomolecular and clinical aspects. 2nd ed. (CRC Press, Boca Raton, FL, 2008)

58. M. Puri, D. Sharma, C.J. Barrow, Trends Biotechnol. 30, 37-44 (2012)

59. A. Scalbert, C. Andres-Lacueva, M. Arita, P. Kroon, C. Manach, M. Urpi-Sarda, D. Wishart, J. Agric. Food Chem. 59, 4331-4348 (2011)

60. Ö, Tokusoglu, C. Hall, Fruit and cereal bioactives: sources, chemistry and applications (CRC Press, Boca Raton, FL, 2011)

61. R.H. Liu, Adv. Nutr. 4, 384S-392S (2013)

62. R. Dhalaria, R. Verma, D. Kumar, S. Puri, A. Tapwal, V. Kumar, E. Nepovimova, K. Kuca, J. Antiox. 9, $1123(2020)$

63. D.S. Ferreira, A.F. Faria, C.R.F Grosso, A.Z. Mercadante, J. Braz. Chem. Soc. 20, 1908-1915 (2009)

64. M. Gonnet, L. Lethuaut, F. Boury, J. Control. Release. 146, 276-290 (2010)

65. J. Gerritsen, F. Crum, Int. Food Res. J. 3, 40-41 (2002)

66. N. Reavley, The encyclopaedia of vitamins, minerals, supplements and herbs (Bookman Press, Victoria, 1998)

67. N. N. Potter, J. H. Hotchkiss, Food Science (Chapman \& Hall, New York, 1995)

68. Y.O. Li, V.P.D. González, L.L. Diosady, Microencapsulation in the food industry. A practical implementation guide (Academic Press, Elsevier, Amsterdam - Boston - Heidelberg - London - New
York - Oxford - Paris - San Diego - San Francisco Singapore - Sydney - Tokyo, 2014)

69. N. Wilson, N. Shah, ASEAN Food J. 14, 1-14 (2007)

70. S. Abbas, C.D. Wei, K. Hayat, Z. Xiaoming, Food Rev. Int. 28, 343-374 (2012)

71. V.B Junyaprasert, A. Mitrevej, N. Sinchaipanid, P. Broome, D.E. Wurster, Drug Dev. Ind. Pharm. 27(6), 561-566 (2001)

72. V. Nedovic, A. Kalusevic, V. Manojlovic, S. Levic, B. Bugarski, Procedia Manuf. 1, 1806-1815 (2011)

73. A. Ramakrishnan, N. Pandit, M. Badgujar, C. Bhaskar, M. Rao, Bioresour. Technol. 98, 368-372 (2007)

74. F. Gassara-Chatti, S.K. Brar, C.M. Ajila, M. Verma, R.D. Tyagi, J.R. Valero, Food Chem. 137, 18-24 (2013)

75. M.E. Sanders, Arch. Clin. Infect. Dis. 46, S58-S61 (2008)

76. P. De Vos, M.M. Faas, M. Spasojevic, J. Sikkema, Int. Dairy J. 20, 292-302 (2010)

77. S. Krishnan, A.C. Kshirsagar, R.S. Singhal, Carbohydr. Polym. 62, 309-315 (2005)

78. Y.D. Kim, C.V. Morr, J. Agric. Food Chem. 44, 13141320 (1996)

79. L.T. Lim, Comprehensive biotechnology (Academic Press, Elsevier, San Diego, 2011)

80. L.T. Lim, Nano- and microencapsulation for foods (Wiley-Blackwell, John Wiley \& Sons Ltd, Hoboken, NJ, 2014)

81. M. Millette, C. Le Tien, W. Smoragiewicz, M. Lacroix, Food Control. 18, 878-884 (2007)

82. L. Vermeiren, F. Devlieghere, J. Debevere, Food Addit. Contam. 19, 163-171 (2002)

83. G. Mauriello, E. De Luca, A. La Storia, F. Villani, D. Ercolini, Lett. Appl. Microbiol. 41, 464-469 (2005)

84. S. Quintavalla, L. Vicini, Meat Sci. 62, 373-380 (2002)

85. M.A. Del Nobile, M. Cannarsi, C. Altieri, M. Sinigaglia, P. Favia, G. Iacoviello, R. D’Agostino, J. Food Sci. 69, 379-383 (2004)

86. N.B. Franklin, K.D. Cooksey, K.J.K. Getty, J. Food Protect. 67, 480-485 (2004)

87. S. Min, T.R. Rumsey, J.M. Krochta, J. Food Eng. 84, 39-47 (2008)

88. A. Conte, M. Sinigaglia, M.A. Del Nobile, J. Food Protect. 69, 861-865 (2006)

89. A.A. Al-Nabulsi, R.A. Holley. J. Appl. Microbiol. 100, 244-255 (2006)

90. K.L. Hoffman, I.Y. Han, P.L. Dawson, J. Food Protect. 64, 885-889 (2001)

91. R.D. Joerger, Packag. Technol. Sci. 20, 231-273 (2007)

92. J.W. Rhim, S.I. Hong, H.M. Park, P.K.W. Ng, J. Agr. Food Chem. 54, 5814-5822 (2006)

93. E. Kristo, K.P. Koutsoumanis, C.G. Biliaderis, Food Hydrocol. 22, 373-386 (2008)

94. E. Ascota, Curr. Opin. Colloid Interf. Sci. 14, 3-15 (2009)

95. P.R. Srinivas, M. Philbert, T.Q. Vu, Q. Huang, J.L. Kokini, E. Saltos, H. Chen, Nutr. J. 140, 119-124 (2010) 
96. Q. Huang, H. Yu, Q. Ru, J. Food Sci. 75, R50-R57 (2010)

97. H.B. Nair, B. Sung, V.R. Yadav, R. Kannappan, M.M. Chaturvedi, B.B. Aggarwal, Biochem. Pharmacol. 80, 1833-1843 (2010)

98. A.G. Gaonkar, N. Vasisht, A.R. Khare, R. Sobel. Microencapsulation in the food industry. a practical implementation guide (Academic Press, Elsevier, Amsterdam - Boston - Heidelberg - London - New York - Oxford - Paris - San Diego - San Francisco Singapore - Sydney - Tokyo, 2014)

99. S.K.B. Vimala, J.A. Moses, C. Anandharamakrishnan, Polymers for food applications (Springer, New York, 2018)

100. M. Arenas-Jal, J.M. Suñé-Negre, P. Pérez-Lozano, E. García-Montoya, Crit. Rev. Food Sci. Nutr. 14, 2405-2421 (2019)

101. C. Radnitz, B. Beezhold, J. DiMatteo, J. Appetite. 90, 31-36 (2015)

102. D. Hancox, The unstoppable rise of veganism: How a fringe movement went mainstream (The Guardian Guardian News \& Media Limited, London, 2018)

103. The Nielsen Company, The Nielsen homescan panel protein survey from April 2017 (NielsenIQ, Ney York, 2017)

104. A. Nesterenko, I. Alric, F. Silvestre, V. Durrieu, Ind. Crops Prod. 42, 469-479 (2013)

105. J.A. Tapia-Hernández, C.L. Del-Toro-Sánchez, F.J. Cinco-Moroyoqui Trends Food Sci. Technol. 90, 111-132 (2019)

106. F. Nazzaro, P. Orlando, F. Fratianni, R. Coppola, Curr. Opin. Biotechnol. 23, 182-186 (2012)

107. B. Magnuson, I. Munro, P. Abbot, Food Addit. Contam. 30, 1147-1220 (2013) 